\title{
The case for quality improvement in emergency medicine
}

\author{
Shawn Mondoux, BEng, MD, MSc*
}

\section{EDITORIAL}

In this article, Chartier et al. succinctly and accurately described the first steps of a quality improvement (QI) initiative within any clinical setting. Their measured and exact approach to the science of QI reflects current best practice and the founding tenets of QI advisers across the spectrum of healthcare. This formulaic method was made more relevant to the field of emergency medicine, in which a multitude of stakeholders and an ongoing battery of pressures on the systems of care exists. The tools described are as versatile as they are ubiquitous within QI, having been applied initially in manufacturing and progressively across many disciplines and within every corner of health care. This series of articles is an exceptional guide for beginners and experts alike on how to best apply QI for targeted, measurable, and patient-centred outcomes.

Although these articles clarify the QI methodology, many questions remain around the necessity of clinical QI activities. Clinicians and health care teams question the track record of so called "quality initiatives," which take place in their clinical environment. Readers may ask, "Why bother doing QI?" Quite simply, the act of delivering medical care has been demonstrated to be the cause of significant patient morbidity and mortality. To be clear, in endeavouring to minimize patient suffering and harm, we have created systems of care in which a significant by-product is suffering and harm. We are fostering that which we are trying to solve. ${ }^{1-4}$ Short of creating adverse outcomes, we have not optimally designed our systems to achieve the best possible results. Despite our attempts, an important proportion of our care does not meet care standards established in the literature. ${ }^{5,6}$ As we have all pledged the Hippocratic oath (Primum non nocere), we cannot consciously plunge into practice knowing that our care systems are, despite their well-intentioned design, the source of suffering for our patients. QI offers us a toolbox and skill set to address these very issues.

An astute critique is that QI is the latest en vogue trend, used to further administrative agendas or to meet provincial benchmarks. The discerning practitioner must be aware that process improvement initiatives that do not place the patient at the centre of improvement and fail to incorporate rigorous improvement methodology cannot be called QI. We cannot see previously failed attempts as evidence of the failure of QI as a science. Rather it is the mislabelling of an initiative that failed to adhere to true QI principles. In those cases, where appropriate QI methodologies are applied, and improvement gains are not achieved, we must consider that these "negative trials" contribute to the collective knowledge regarding problems we are attempting to solve. If well-conducted epidemiological or clinical trials publish a negative result, we do not conclude that the field of clinical epidemiology is worthless. While considering the science of QI, we must be cautious not to "throw the baby out with the bathwater." A reasonable solution is to focus on providing emergency medicine practitioners with the tools to appraise critically the rigour of QI work and make judgments based on methodology rather than outcomes.

A secondary critique of QI science often includes the observation that many improvements are not sustained in the long-term. Indeed, sustaining improvement is the most underestimated phase of a QI intervention. ${ }^{7}$ Many published papers focusing on successful short-term change have not engaged in a parallel process of culture change in the clinical setting or built-in a

From the *Department of Medicine, Division of Emergency Medicine, McMaster University, Hamilton, ON.

Correspondence to: Shawn Mondoux, 237 Barton St E, McMaster Clinics, Room 250, Hamilton, ON L8L2X2; Email: shawn.mondoux@medportal.ca 
sustainability plan from the outset of the project. Once the positive change is observed, subsequent Plan-DoStudy-Act cycles must be devoted to sustaining results after the improvement team has folded. This includes ongoing and regular data monitoring, forced-function process changes, and policy modifications where applicable. Although the critique of sustainability is fair, it is not unique to QI. If QI had demonstrated itself to be the cure-all to habit, culture, and heterogeneous behaviour, it would have become a core part of health care long ago. Instead of relegating the skill of culture change to our leaders, QI gives people on the ground tools to groom an environment for more successful culture change.

Lastly, to address our internal culture element, emergency physicians (EPs) may simply ask, "Why should I care?" This may be the most important question to address. In considering extrinsic motivations, a regulatory shift is occurring in many provinces to emphasize the quality of care provided rather than volumes of care alone. ${ }^{8-11}$ As data are gathered and health care dollars become more scarce, it is reasonable to consider that provincial and federal governments might begin to tie increasingly large volumes of emergency department funding to performance measured by objective quality indicators. Should this occur, the demand for physicians with QI training will dramatically increase and, when reconciled with the QI literature around current QI physician capacity, ${ }^{12}$ will create a demand that dwarfs supply. Despite this probability of future need, recognition of QI work for involved EPs remains limited. Academic promotion streams for "clinician improvers" exist at very few institutions. QI clinicians cannot compete in investigator streams as grant pools are smaller. There are relatively few dedicated QI peer-reviewed journals. Our non-academic colleagues may thus have little incentive to change their practice environment for the better.

Yet, herein lies the ultimate power and lever of QI: to provide the emergency medicine (EM) practitioner with the tools to change their practice environment for the best. When improvement advisers search for quality problems to solve, they often ask "What is the most annoying part of the patient and clinician experience in your clinical setting?" QI is fundamentally an empowerment tool that creates a community of individuals assembled around a clear well-articulated clinical problem with the goal of improving the patient experience. To the interested few, we must make available progressive tiers of education in this specialized skill set.
Participation in QI remains a highly personal costbenefit calculation. Without clear incentives outside continuing medical education (CME) credits and publication, many will choose not to participate. Despite this, others, i.e., early adopters, will want to explore QI as a field, and we should make opportunities available to our colleagues who may eventually make the long sought-after changes in their practice environment.

We cannot shirk our role as influencers in the delivery of health care as we are an essential component of all QI initiatives. The first step is admitting there is a problem: that the process of delivering of health care might contribute as much to outcomes as the therapies provided. To this end, we must agree that carefully calculated process experimentation in the clinical setting may have the greatest potential to change the patient experience and the quality of care we provide.

\section{Competing interests: None to declare.}

Keywords: quality, quality improvement, patient safety, quality care, emergency medicine, emergency department

\section{REFERENCES}

1. Kohn L, Corrigan J, Donaldson M. To Err Is Human: Building a Safer Health System. Committee on Quality of Health Care in America I of M. Washington, DC: National Academy Press; 2000. Available at: http://scholar.google. $\mathrm{fr} / \mathrm{scholar}$ ? cluster $=15282385942323833969 \& \mathrm{hl}=\mathrm{fr} \& \mathrm{as}$ $\mathrm{sdt}=0,5 \# 1$.

2. Baker GR, Norton PG, Flintoft V, et al. The Canadian Adverse Events Study: the incidence of adverse events among hospital patients in Canada. CMA7 2004;170(11):1678-86.

3. Matlow AG, Baker GR, Flintoft V, et al. Adverse events among children in Canadian hospitals: the Canadian Paediatric Adverse Events Study. CMA7 2012;184(13):E709-18.

4. Makary MA, Daniel M. Medical error-the third leading cause of death in the US. BMF 2016;353:12139.

5. McGlynn EA, Asch SM, Adams J, et al. The quality of health care delivered to adults in the United States. $N$ Engl 7 Med 2003;348(19):2635-45. doi:10.1056/NEJM2003 11063491916.

6. Mangione-Smith R, DeCristofaro AH, Setodji CM, et al. The quality of ambulatory care delivered to children in the United States. N Engl 7 Med 2007;357(15):1515-23. Available at: http://www.ncbi.nlm.nih.gov/pubmed/12826639.

7. Scoville R, Little K, Rakover J, Luther K, Mate K. Sustaining improvement. IHI White Paper. Cambridge: The Institute of Health Care Improvement; 2016.

8. Government of Ontario Ministry of Health and Long-Term Care. Quality Based Procedures. Available at: http://www. health.gov.on.ca/en/pro/programs/ecfa/funding/hs_funding qbp.aspx (accessed April 26, 2017). 
9. Health Quality Ontario. The ED Return Visit Quality Program Available at:http://www.hqontario.ca/portals/0/ documents/qi/introductory-guidance-en.pdf\%0D (accessed April 26, 2017).

10. Health Quality Ontario. Quality Improvement. Available at: http://www.hqontario.ca/Quality-Improvement(accessed April 26, 2017).
11. Health Council of Canada. Which Way to Quality? Key Perspectives on Quality Improvement in Canadian Health Care Systems. Toronto: Health Council of Canada; 2013.

12. Wong BM, Levinson W, Shojania KG. Quality improvement in medical education: current state and future directions. Med Educ 2012;46(1):1071-9. 